\title{
AS22-001. SCREENING FOR COGNITIVE IMPAIRMENT WITH THE MONTREAL COGNITIVE ASSESSMENT IN SPANISH PATIENTS WITH MINOR STROKE OR TRANSIENT ISCHAEMIC ATTACK: A VALIDATION STUDY.
}

J.M. Ramirez-Moreno1, P. Muñoz-Vega ${ }^{2}$, S. Bartolome-Alberca ${ }^{3}$, E. Guerrero ${ }^{4}$, A.M. Roa ${ }^{3}$, A.B. Constantino ${ }^{3}$, J.J. Aguirre ${ }^{1}$. 1. Extremadura University Medical School, Biomedical Sciences, Badajoz, Spain. 2. Neurorehabilitation Center "Casaverde", Neurology area, Mérida, Spain. 3. Hospital Universitario Infanta Cristina, Neurology, Badajoz, Spain. 4. Extremadura University, Psychology and Social Anthropology Department, Badajoz, Spain.

Background and Aims: The symptoms of a minor stroke and transient ischemic attack (TIA) are temporary and mild. Despite the transient nature of focal symptoms and the absence of visible brain lesions in some patients, many experience persistent cognitive problems afterward. We aimed to establish the cut-off point of the Montreal Cognitive Assessment (MoCA) in screening for cognitive impairment within 90 days of TIA.

Methods: A total of 50 acute mild ischaemic stroke and TIA patients were recruited. They received the MoCA test and a formal neuropsychological evaluation. The cognitive impairment was defined clinically by a formal neuropsychological test battery (NEURONORMA). The Neuronorma Battery includes the selection of frequently used tests that covers the main domains of cognitive abilities: attention, work memory, mental control, psychomotor speed, language, visuoperceptive and constructive capacities, memory and executive functions.

The Montreal Cognitive Assessment (MoCA) is a brief 30-question test that takes around 10-12 minutes to complete. Scores on the MoCA range from zero to 30, with a score of 26 and higher generally considered normal. The scoring breakdown is as follows: Visuospatial and Executive Functioning: 5 points; Animal Naming: 3 points; Attention: 6 points; Language: 3 points; Abstraction: 2 points; Delayed Recall (Short-term Memory): 5 points; Orientation: 6 points. Education Level: 1 point is added to the test-taker's score if he or she has 12 years or less of formal education.

Table 2. Score of the domains of the MoCA test in the total of patients with minor stroke and TIA, according to the presence or absence of cognitive impairment based on the neuropsychological examination

\begin{tabular}{|l|c|c|c|c|c|}
\hline Domains & Scoring & $\begin{array}{r}\text { Total } \\
\mathbf{N ~ ( \% )}\end{array}$ & $\begin{array}{c}\mathbf{N C l} \\
\mathbf{N ~ ( \% )}\end{array}$ & $\begin{array}{c}\mathbf{C l} \\
\mathbf{N}(\%)\end{array}$ & $\mathbf{p}$ \\
\hline Visuospatial and Executive & 5 & $4,0(1,1)$ & $4,7(0,6)$ & $3,8(1,1)$ & 0,001 \\
Animal Naming & 3 & $2,9(0,3)$ & $3,0(0,0)$ & $2,9(0,3)$ & 0,044 \\
Attention & 6 & $4,9(1,4)$ & $5,5(0,8)$ & $4,7(1,5)$ & 0,018 \\
Language & 3 & $2,2(0,7)$ & $2,6(0,5)$ & $2,1(0,7)$ & 0,030 \\
Abstraction & 2 & $0,8(0,8)$ & $1,1(0,8)$ & $0,8(0,7)$ & 0,233 \\
Delayed Recall & 5 & $1,8(1,7)$ & $2,7(1,7)$ & $1,5(1,6)$ & 0,026 \\
Orientation & 6 & $5,8(0,6)$ & $5,7(0,8)$ & $5,8(0,4)$ & 0,435
\end{tabular}

Table 3. Discriminant indices of MoCA in detecting cognitive impairment at 90 days in patients with minor stroke and TIA.

\begin{tabular}{lcccccc} 
Cuttoff score & Se (\%) & Sp (\%) & PPV(\%) & NPV (\%) & LR + & LR- \\
\hline$<=23,00$ & 37,8 & 100,0 & 100,0 & 36,1 &. & 1,61 \\
$<=24,00$ & 56,8 & 92,3 & 95,5 & 42,9 & 7,38 & 2,13 \\
$<=25,00^{*}$ & 78,4 & 76,9 & 90,6 & 55,6 & 3,40 & 3,56 \\
$<=26,00$ & 83,8 & 53,8 & 83,8 & 53,8 & 1,82 & 3,32 \\
$<=27,00$ & 91,9 & 38,5 & 81,0 & 62,5 & 1,49 & 4,74 \\
$<=28,00$ & 97,3 & 23,1 & 78,3 & 75,0 & 1,26 & 8,54 \\
NPV, negative predictive value; PPV, positive predictive value; Se, sensitivity; Sp, specificity. & & &
\end{tabular}

Results: The average age of recruited patients was $57.7 \pm 8.0$ years, with the majority of patients being men (70.0\%) and all patients with greater equal or than primary school level of education. Thirty-seven (74.0\%) subjects were classified into Cognitive impairment $(\mathrm{Cl})$ group. The optimal cut-off point for MoCA in discriminating patients with $\mathrm{Cl}$ from those with no cognitive impairment was 25 (sensitivity 78,4\% (IC95\%: 62,8 - 88,6\%), specificity 76,9\% IC95\%: 49,7 - 91,8\%), positive predictive value 90,6\% (IC95\%: 81,0 - 95,6\%), negative predictive value 55,6\% (IC95\%: 39,5 - 70,4\%), and the Receiver operating characteristic curve analysis (AUC) $0.835(95 \% \mathrm{Cl} 0.720-0.949)$.

Table. Clinical characteristics of patients with acute mild stroke or TIA.

\begin{tabular}{|c|c|c|c|c|}
\hline & $\begin{array}{l}\text { Total } \\
\mathrm{N}=50\end{array}$ & $\begin{array}{c}\text { No cognitive } \\
\text { impairment } \\
n=13\end{array}$ & $\begin{array}{c}\text { Cognitive } \\
\text { impairment } \\
n=37\end{array}$ & $p$ \\
\hline Sex, men (\%) & $35(70)$ & $10(76,9)$ & $25(67,6)$ & 0,527 \\
\hline Age (year), mean (SD) & $57,7(8,0)$ & $57,3(7,3)$ & $57,8(8,3)$ & 0,851 \\
\hline Residence urban (\%) & $28(56)$ & $10(76,9)$ & $18(48,6)$ & 0,077 \\
\hline \multicolumn{5}{|l|}{ Education } \\
\hline - Primary school and below (\%) & $25(50)$ & $6(46,2)$ & $19(51,4)$ & 0,926 \\
\hline - Middle and high school (\%) & $22(44)$ & $6(46,2)$ & $16(43,2)$ & \\
\hline - Bachelor and above (\%) & $3(6)$ & $1(7,7)$ & $2(5,4)$ & \\
\hline \multicolumn{5}{|l|}{ Etiology } \\
\hline - atherosclerosis (\%) & $9(18)$ & $1(7,7)$ & $8(21,6)$ & 0,247 \\
\hline - cardioembolism (\%) & $4(8)$ & $0(0,0)$ & $4(10,8)$ & \\
\hline - Small artery occlusion (\%) & $12(24)$ & $5(38,5)$ & $7(18,9)$ & \\
\hline - undetermined(\%) & $22(44)$ & $7(53,8)$ & $15(40,5)$ & \\
\hline - other (\%) & $3(6)$ & $0(0,0)$ & $3(8,1)$ & \\
\hline \multicolumn{5}{|l|}{ Medical history } \\
\hline Number of risk factors, mean (SD) & $3,4(1,7)$ & $3,2(2,4)$ & $3,4(1,5)$ & 0,709 \\
\hline Hypertension (\%) & $29(58)$ & $6(46,2)$ & $23(62,2)$ & 0,314 \\
\hline Diabetes (\%) & $14(28)$ & $5(38,5)$ & $9(24,3)$ & 0,329 \\
\hline Hyperlipidaemia (\%) & $19(38)$ & $5(38,5)$ & $14(37,8)$ & 0.968 \\
\hline Atrial firbillation (\%) & $4(8)$ & $0(0,0)$ & $4(10,8)$ & 0,216 \\
\hline Coronary heart disease (\%) & $5(10)$ & $1(7,7)$ & $4(10,8)$ & 0,747 \\
\hline Smoking (\%) & $30(60)$ & $8(61,5)$ & $22(61,1)$ & 0,978 \\
\hline Alcohol (\%) & $20(40,8)$ & $7(53,8)$ & $13(36,1)$ & 0,265 \\
\hline Depresion (\%) & $1(2)$ & $1(7,7)$ & $0(0,0)$ & 0,260 \\
\hline Family history of stroke (\%) & $14(28)$ & $5(38,5)$ & $9(24,3)$ & 0,474 \\
\hline Family history of dementia (\%) & $13(26)$ & $3(33,3)$ & $9(24,3)$ & 0,539 \\
\hline \multicolumn{5}{|l|}{ Neuroimage } \\
\hline DWI positive (\%) & $42(84)$ & $9(62,2)$ & $33(89,2)$ & 0,231 \\
\hline
\end{tabular}

Figure. Receiver operating characteristic (ROC) analysis of Montreal Cognitive Assessment (MoCA) for differentiating patients with cognitive impairment from patients without cognitive impairment (Se 78,4\%, Sp $\mathbf{7 6 , 9 \%}$, area under curve $=$ 0.835).

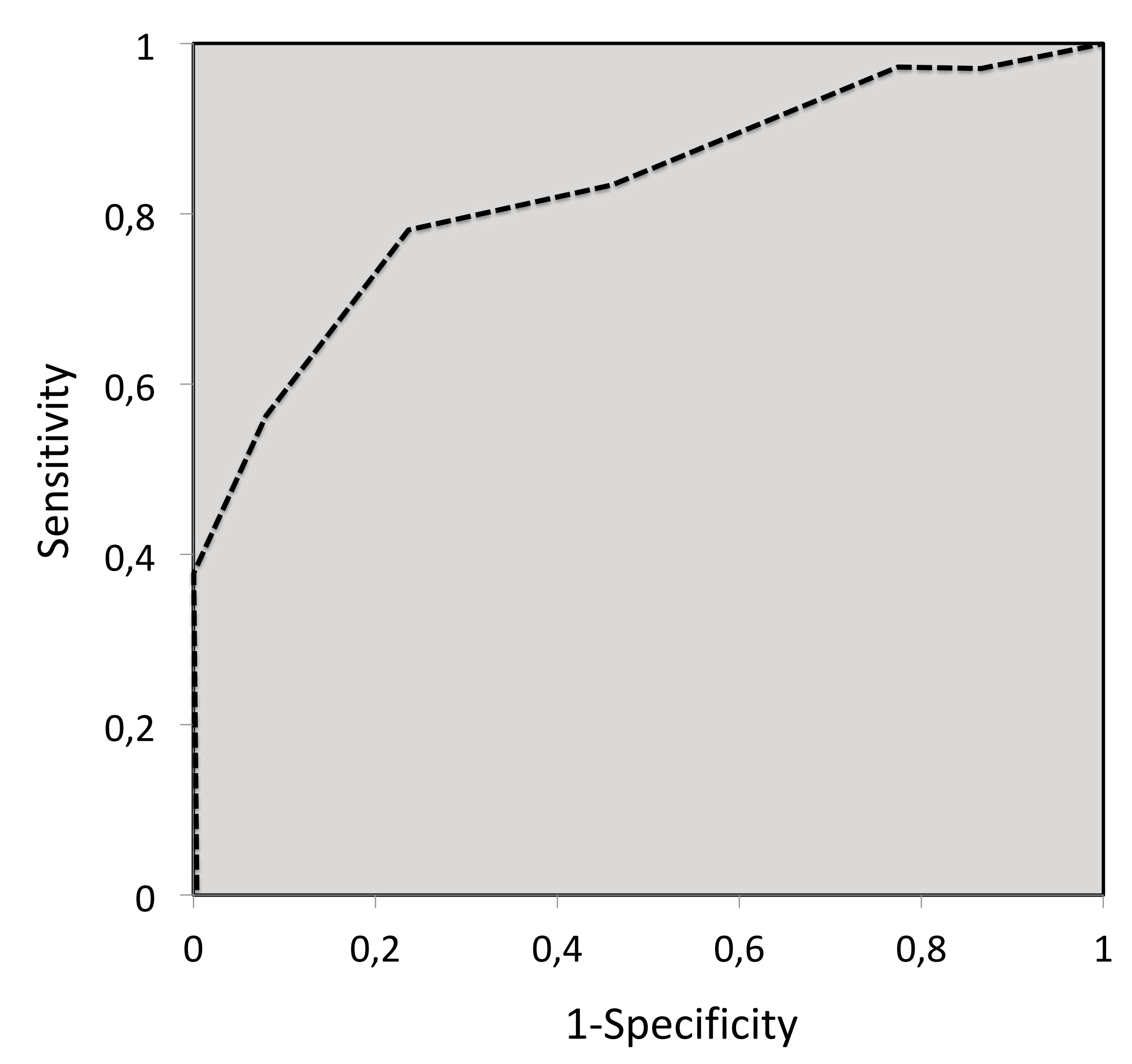

Conclusions: More than half of the sample had cognitive impairment as determined by the formal battery of neuropsychological tests. A MoCA cut-off score of 25 is optimally sensitive and specific for detecting $\mathbf{M C l}$ after mild stroke or TIA in the subacute stroke phase. Therefore, it should be implemented in routine clinical practice. 